\title{
Advances in the Analysis of Topographic Features on Discrete Images
}

\author{
Pierre Soille \\ EC Joint Research Centre \\ Institute for Environment and Sustainability \\ TP 262, I-21020 Ispra, Italy \\ Pierre.Soille@jrc.it
}

\begin{abstract}
By viewing the grey scale values of 2-dimensional (2-D) images as elevation values above the image definition domain, geomorphological terms such as crest lines, watersheds, catchment basins, valleys, and plateaus have long been used in digital image processing for referring to image features useful for image analysis tasks. Because mathematical morphology relies on a topographic representation of 2-D images allowing for grey scale images to be viewed as 3-D sets, it naturally offers a wide variety of transformations for extracting topographic features. This paper presents some advances related to the imposition of minima, the lower complete transformation, the hit-or-miss transform, and the extraction of crest lines by a skeletonisation procedure.
\end{abstract}

Keywords. Mathematical morphology, minima imposition, plateau, lower complete transformation, grey scale hit-or-miss, crest lines, grey scale skeletonisation, watersheds

\section{Introduction}

The topographic representation of grey scale images has long been considered in digital image processing for extracting features such as ridges and valleys [11], watersheds [8], and plateaus. In contrast to approaches based on the analysis of the discrete derivatives of the image, mathematical morphology [20] offers a set theoretic framework which can be considered as a suitable alternative approach in some applications. In this paper, we adopt the morphological framework and revisit several transformations dealing with the processing of topographic features of digital 2-dimensional (2-D) grey scale images. Background definitions and notations used in this paper are described in [26].

In section 2 we introduce a minima imposition technique based on a carving procedure rather than the usual reconstruction based technique. This approach is a suitable alternative in situations where the creation of flat regions by the reconstruction would impede further processing. We then show in section 3 that the suppression of image plateaus can be achieved by an interpolation technique taking into account the morphology of both the descending and ascending plateau

^ This work was supported by the EC-JRC EuroLandscape Project.

A. Braquelaire, J.-O. Lachaud, and A. Vialard (Eds.): DGCI 2002, LNCS 2301, pp. 175-186 2002. (C) Springer-Verlag Berlin Heidelberg 2002 
borders. This is enabled by a grey weighted geodesic distance called the geodesic time function. The proposed approach leads to an enhanced algorithm for the lower complete transformation which a necessary preprocessing for computing the steepest slope path linking an arbitrary pixel to the regional minimum of its corresponding catchment basin. In section 4, we extend the hit-or-miss transform to the processing of grey scale images by introducing the notion of constrained and unconstrained hit-or-miss. These operations are useful for extracting specific grey scale neighbourhood configurations. Before concluding, we show in section 5 that crest lines can be obtained by order independent homotopic thinnings.

\section{Minima Imposition by Carving}

The minima imposition is an image transformation which modifies an input grey scale image in such a way that the only remaining minima are those given by an additional input image called marker image. Note that we refer to an image minimum in the sense of a regional minimum, i.e., a connected component of pixels having the same value and whose external boundary pixels have all a greater value. Denoting by $f$ the input grey scale image, the marker image $f_{m}$ is defined as follows: $f_{m}(\mathbf{x})=0$ if $\mathbf{x}$ belongs to a marker, $t_{\max }$ otherwise. The minima imposition is usually achieved by performing the following morphological reconstruction by erosion $R^{\varepsilon}[2,14]: R_{(f+1) \wedge f_{m}}^{\varepsilon}\left(f_{m}\right)$. This procedure is a connected 1 algebraic closing which may create large flat regions because the catchment basins of all unmarked minima are partially filled in. This is not a problem if one is interested in computing the watersheds of the filtered image. However, there are applications where markers of thin nets can be detected while a watershed could not be used for extracting the nets due to gaps occurring along the net. Indeed, in this situation, the reconstruction based minima imposition removes all parts of the net which do not contain a marker. In addition, if one would like to simulate a flow of water on the topographic representation of the image, the reconstruction based minima imposition has the drawback of creating potentially large flat regions which in turn pose a problem for the determination of flow directions from a local neighbourhood. These problems motivated us to look for an alternative way to impose the minima of an image.

Rather than filling the unmarked regional minima, we propose to carve the image in such a way that these regional minima flow further down. The carving procedure relies also on a flooding simulation but, rather then filling in unmarked minima, a non-ascending path linking them to their nearest marked minimum is created. The pseudo-metric used for calculating the distance between an unmarked minimum and a marked minimum is based on the flooding paths. The procedure is detailed hereafter.

First, all regional minima not marked by the marker image are stored in a binary image. The flooding simulation then starts by inserting into a priority

\footnotetext{
${ }^{1}$ An operator is connected $[21,19]$ if and only if it coarsens the partition of any given input image, the partitioning being taken in the sense of that induced by the flat zones of the image.
} 
queue the external boundary pixels of the marker minima, the priority being inversely proportional to the intensity value of the considered pixel. Pixels are then iteratively retrieved from the non-empty queue with the highest priority (i.e., lowest intensity elevation) while inserting their unprocessed neighbours in the priority queue (again considering a priority inversely proportional to their elevation). An additional image is used to store the direction of propagation of the flood at each pixel. Before inserting a pixel in the queue, we check whether it belongs to an irrelevant minimum. If so, the stored directions are used to backtrack the flooding path until we reach a pixel of elevation equal to that of the reached minimum and set all pixels along the path to this elevation. The reached minimum is then discarded from the binary mask of irrelevant minima while inserting all its unprocessed external boundary pixels in the priority queue. The process terminates when the priority queue is empty. By construction, all irrelevant minima are removed by the procedure because each of them can be linked to a marker by a non-ascending path.

A comparison between the minima imposition by reconstruction and by carving is performed in Fig. 1 on two samples of a Pan-European digital elevation model (DEM). Given the scale of the input DEM $(250 \mathrm{~m})$, a valid assumption is to consider that all minima occurring inside the DEM definition domain are irrelevant because almost all European catchment basins are connected to the sea. Therefore the fillhole transformation generalised to grey scale images by a reconstruction by erosion can be used to suppress all interior minima as originally proposed in [29]: $\operatorname{FILL}(f)=R_{f}^{\varepsilon}\left(f_{m}\right)$, where $f_{m}(\mathbf{x})=f(\mathbf{x})$ if $\mathbf{x}$ lies on the border of $f, \max (f)$ otherwise. Figures 1(a)-(b) illustrate that the reconstruction based minima imposition fills most of the fragments of the stream so that any subsequent flow simulation process will be unable to follow the stream path indicated by lower elevation values in the initial data. Figures. 1(d)-(e) illustrate another problem occurring when a stream goes through a very narrow valley. Indeed, if it happens that the DEM resolution is too coarse to resolve a narrow valley, the fillhole procedure will fill it upstream creating thereby a large flat region suppressing relevant topography in the valley bottom. The images appearing at the bottom of Fig. 1 show that both problems are solved by the carving procedure.

Contrary to the minima imposition by reconstruction, the carving procedure is not a connected algebraic closing but merely an anti-extensive and idempotent operation.

In contrast to the flooding simulation introduced in $[5,34]$ for the computation of watershed boundaries and [12] for the computation of drainage networks, the proposed carving algorithm does not require the prior sorting of the image pixels in increasing order of elevation. Indeed, the sorting is achieved by the priority queue which is initialized by the relevant image minima. A similar approach has already been used for the computation of watersheds [3] and contributing drainage areas [30]. As concerns the computation of the binary mask of all regional minima, it is readily obtained thanks to the fast algorithm proposed by Breen and Jones [4], see also [26, p. 169]. 

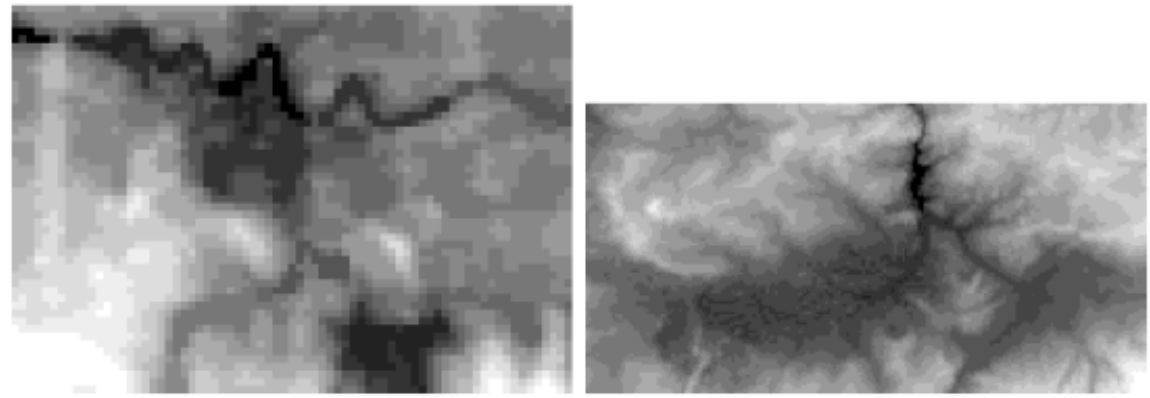

(a) $73 \times 51$ sample of a Pan-European DEM at the (d) Another sample $(211 \times 110$ pixels $)$. spatial resolution of $250 \mathrm{~m}$.
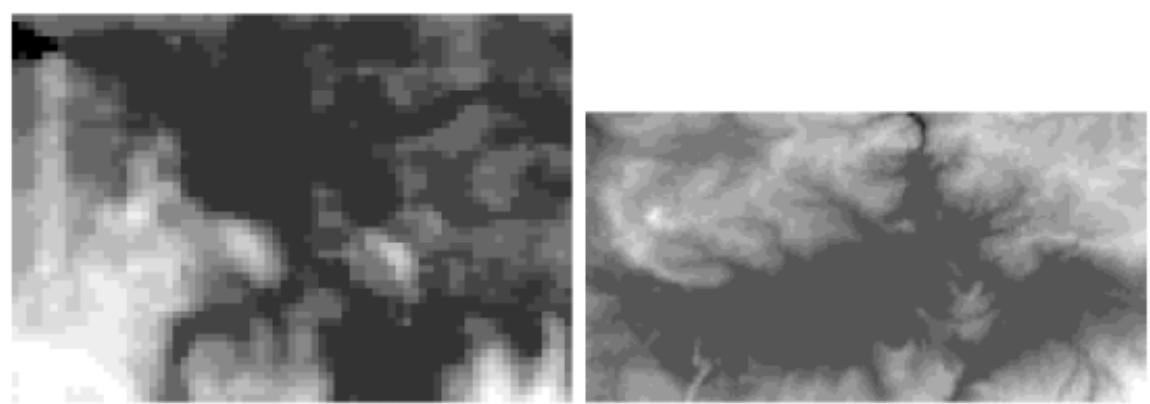

(b) Same sample as in (a), after minima imposi- (e) Same sample as in (d), after minima imposition tion based on reconstruction by erosion. based on reconstruction by erosion.
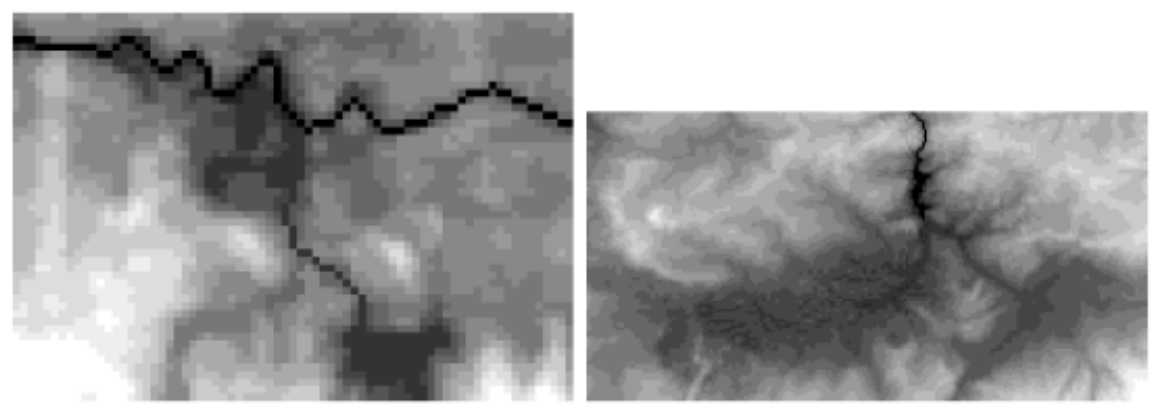

(c) Same sample as in (a), after minima imposition (f) Same sample as in (c), after minima imposition based on carving. based on carving.

Fig. 1. Minima imposition: Comparison between procedures based on morphological reconstruction by erosion and the proposed carving. The displayed samples were extracted from a Pan-European DEM at the resolution of $250 \mathrm{~m}$. The markers were defined for both procedures as the regional minima of the input DEM connected to its border, according to [29]. 


\section{Lower Complete Transformation}

In some applications, it may be useful to transform an input image into a lower complete image, i.e., an image where the only pixels having no neighbour with a strictly lower intensity are those belonging to the regional minima of the image [13]: $f$ is lower complete if and only if $\forall p \notin \operatorname{RMIN}(f), \exists p^{\prime} \in N_{\mathcal{G}}(p) \mid f\left(p^{\prime}\right)<$ $f(p)$. For example, a lower complete image is necessary for calculating the steepest slope path linking a pixel to a regional minimum. Indeed, steepest slope directions can be calculated for each pixel of a lower complete image. Another potential application is for watershed transformation because, on a lower complete image, the calculation of the distance function on plateaus advocated in [34] can be skipped.

An image can be transformed into a lower complete image by adding an auxiliary relief to the plateaus. This relief is defined as the geodesic distance function calculated from the descending border of the plateau (reference set) using the plateaus as geodesic mask. A fast implementation based on priority queues is detailed in [30]. However, this procedure tends to create parallel flow directions. Garbrecht and Martz [9] proposed an alternative procedure ensuring better flow convergence whereby the topography on the plateau is created by adding the inverse geodesic distance from higher terrain to the geodesic distance from lower terrain may itself contain a plateau. However, as noted by these authors, the topography created on the plateau may itself contain a plateau. A solution to this problem is to define the topography on the plateau as the geodesic time function $[23,25]$ using the descending border of the plateaus as marker and the inverse of the geodesic distance from higher terrain as grey scale geodesic mask (see also [24]). This procedure is illustrated in Fig. 2 for a $7 \times 7$ image containing a plateau. Figure $2 \mathrm{~b}$ shows the inverse geodesic distance function computed on the plateau pixels having no lower neighbour and starting from the neighbour pixels having a higher elevation. However, contrary to the methodology described by Garbrecht and Martz [9], we do not compute the geodesic distance away from lower elevations but the geodesic time function [25] using the inverse of the geodesic distance (Fig. 2b) as geodesic mask and the descending border as marker image. The resulting geodesic time function is displayed in Fig. 2r. By construction, a flow direction is directly defined for all plateau pixels as illustrated in Fig. $2 \mathrm{~d}$. The flow direction of a given point is set as the direction of its 8-nearest neighbour point producing the steepest downward slope.

As concerns the computation load, fast algorithms for geodesic distance functions are described in [33] for 8-connected and [22] for Euclidean distance calculations. A fast geodesic time function algorithm based on priority queues is described in [26, pp. 202-202].

\section{Extraction of Surface Specific Neighbourhoods}

On a binary image, the extraction of specific neighbourhood configurations is based on the hit-or-miss transform: The hit-or-miss transformation, $H M T$, of a 


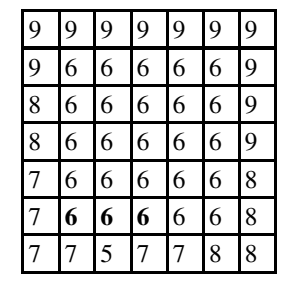

(a) Input image with plateation 6 .
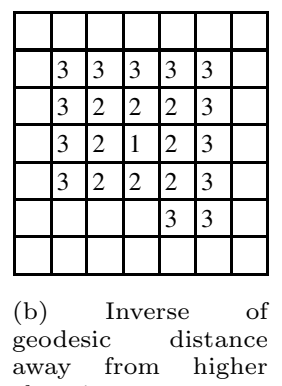
away from higher elevations.

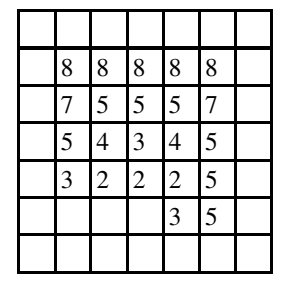

(c) Created topography using geodesic time function.

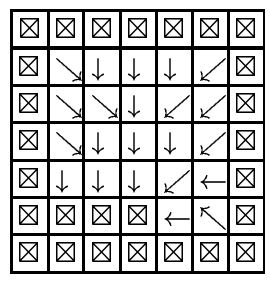

(d) Resulting flow directions.

Fig. 2. Determination of flow directions on a plateau from an artificial topography created by computing the geodesic time function from the descending border of the plateau (plateau pixels in bold) using the inverse of the geodesic distance away from higher elevations as geodesic mask. In the image of flow directions (d), the flow direction of pixels marked by the $\nabla$ sign is set according to the original values of the image.

set $X$ by a composite structuring element $\mathbf{B}=\left(B_{F G}, B_{B G}\right)$ is the set of points, $\mathbf{x}$, such that when the origin of $\mathbf{B}$ coincides with $\mathbf{x}, B_{F G}$ fits $X$ and $B_{B G}$ fits $X^{c}$ :

$$
H M T_{\mathbf{B}}(X)=\left\{\mathbf{x} \mid\left(B_{F G}\right)_{\mathbf{x}} \subseteq X,\left(B_{B G}\right)_{\mathbf{x}} \subseteq X^{c}\right\}=\varepsilon_{B_{F G}}(X) \cap \varepsilon_{B_{B G}}\left(X^{c}\right) .
$$

This equation could be extended to grey scale images but in this latter case we would combine the erosion of a grey scale image with an erosion of the complement of this image. In addition, due to the increasingness of the HMT, the stacking of the hit-or-miss transformations of the successive cross-sections of a grey scale image does not define the subgraph of a grey scale image. However, as illustrated in Fig. [3 for a 1-D signal, when we position $\mathbf{B}$ at a given pixel $\mathbf{x}$, $B_{F G}$ matches the cross-sections of $f$ from the level 0 up to a given level which we denote by $t_{F G}$, while $B_{B G}$ matches the complement of the cross-sections of $f$ from the level $t_{\max }+1$ down to a given level which we denote by $t_{B G}$. For example, in Fig. 3 3 at position $x=8, B_{F G}$ matches the cross-sections of $f$ from the level 0 up to the level $t_{F G}=5$ while $B_{B G}$ matches the complement of the cross-sections of $f$ from the level $t_{\max }$ down to the level $t_{B G}=3$.

Depending on whether we constrain the SE component containing the origin to match either the foreground (if $O \in B_{F G}$ ) or the background (if $O \in B_{B G}$ ) of $\mathbf{x}$, we will obtain two different definitions for the grey tone hit-or-miss transform. The first will be referred to as the unconstrained hit-or-miss. Accordingly, the previously mentioned constraint is at the basis of the constrained hit-or-miss.

Unconstrained hit-or-miss. The output of the unconstrained hit-or-miss, denoted by $U H M T$, of a grey scale image $f$ by a composite SE $\mathbf{B}$ at a position $\mathbf{x}$ is defined as the number of cross-sections $C S_{t}(f)$ such that $B_{F G}$ at $\mathbf{x}$ matches $C S_{t}(f)$ while, simultaneously, $B_{B G}$ at $\mathbf{x}$ matches $\complement C S_{t}(f)$ :

$$
\left[U H M T_{\mathbf{B}}(f)\right](\mathbf{x})=\operatorname{card}\left\{t \mid\left(B_{F G}\right)_{\mathbf{x}} \subseteq C S_{t}(f),\left(B_{B G}\right)_{\mathbf{x}} \subseteq \complement C S_{t}(f)\right\} .
$$




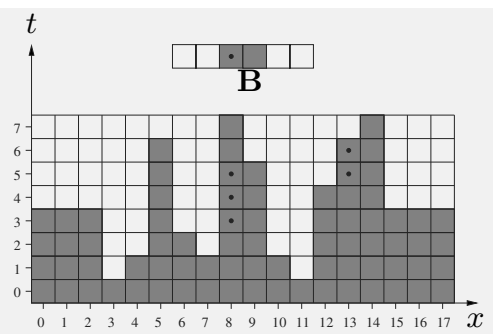

(a) SE $\mathbf{B}$ and input signal $f$ with its subgraph highlighted in grey.

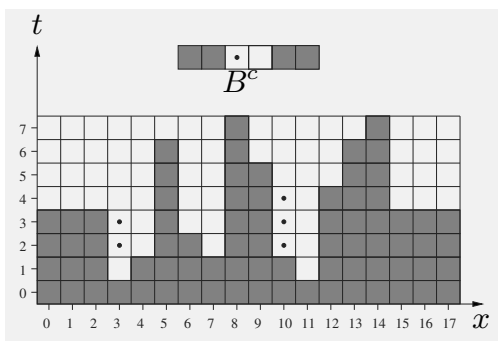

(b) $\mathrm{SE} \mathbf{B}^{c}$ and input signal $f$ with its subgraph highlighted in grey.

Fig. 3. On the extension of the hit-or-miss operator to grey tone images: Two case studies depending on whether the origin of the composite $\mathrm{SE}$ belongs to $B_{F G}$ or $B_{B G}$. In both diagrams, the centre of the pixels of each cross-section (or its complement) matched by the considered composite SE are marked with a bullet.

The unconstrained hit-or-miss transform is illustrated on a 2-D image in Fig. 4.

Constrained hit-or-miss. The definition of the constrained hit-or-miss, denoted by $C H M T$, involves an additional constrain, considered for each pixel position $\mathbf{x}$. Namely, the SE component containing the origin $O$ must match the foreground $\overline{F G}(\mathbf{x})$ if $O \in B_{F G}$ or the background $\overline{B G}(\mathbf{x})$ if $O \in B_{B G}$ (where $\overline{F G}(\mathbf{x})=T_{t \geq f(\mathbf{x})}(f)$ and $\left.\overline{B G}(\mathbf{x})=T_{t \leq f(\mathbf{x})}(f)\right)$ :

$$
\left\{\begin{array}{l}
\left(B_{F G}\right)_{\mathbf{x}} \subseteq \overline{F G}(\mathbf{x}), \text { if } O \in B_{F G} . \\
\left(B_{B G}\right)_{\mathbf{x}} \subseteq \overline{B G}(\mathbf{x}), \text { if } O \in B_{B G} .
\end{array}\right.
$$

As for a graphical representation and looking back to Fig. 3, we only consider the $t$-connected component: 2 of pixels marked by a bullet and which have a nonempty intersection with the $t$-boundary of the subgraph of $f$. This happens for $x=13$ in Fig. 3 a and $x=10$ in Fig. 3 b. In terms of morphological transformations, when $O \in B_{F G},\left(B_{F G}\right)_{\mathbf{x}} \subseteq \overline{F G}(\mathbf{x})$ iff $f(\mathbf{x})=\left[\varepsilon_{B_{F G}}(f)\right](\mathbf{x})$. Accordingly, when $O \in B_{B G},\left(B_{B G}\right)_{\mathbf{x}} \subseteq \overline{B G}(\mathbf{x})$ iff $f(\mathbf{x})=\left[\delta_{B_{B G}}(f)\right](\mathbf{x})$.

Both definitions of the grey tone hit-or-miss are equivalent in the binary case and come down to Eq. 1. In the grey scale case, they are equivalent only in situations where the component of $\mathbf{B}$ containing the origin is restricted to a single pixel. The constrained hit-or-miss is by definition more restrictive than the unconstrained one as highlighted by the following ordering relationship: $C H M T_{\mathbf{B}} \leq U H M T_{\mathbf{B}}$. The hit-or-miss (whether unconstrained or constrained) by $\mathbf{B}$ is complementary to that by $\mathbf{B}^{c}: H M T_{\mathbf{B}}=H M T_{\mathbf{B}^{c}} \complement$. Both types of $H M T$ are non-increasing transformations. From a computational point of view, it can be shown that the proposed HMTs can be defined by performing simple conditional tests on the grey scale erosion by $B_{F G}$ and dilation by $B_{B G}$. In addition,

\footnotetext{
${ }^{2}$ We call $t$-connectivity the $1-\mathrm{D}$ dimensional connectivity defined along the discrete $t$-axis for a fixed value of $x$, i.e., $\left(\mathbf{x}_{1}, t_{1}\right)$ is $t$-connected to $\left(\mathbf{x}_{2}, t_{2}\right)$ iff $\mathbf{x}_{1}=\mathbf{x}_{2}$ and $\left|t_{1}-t_{2}\right|=1$.
} 


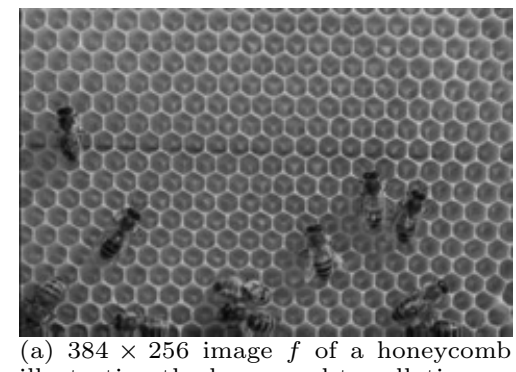

illustrating the hexagonal tessellation.

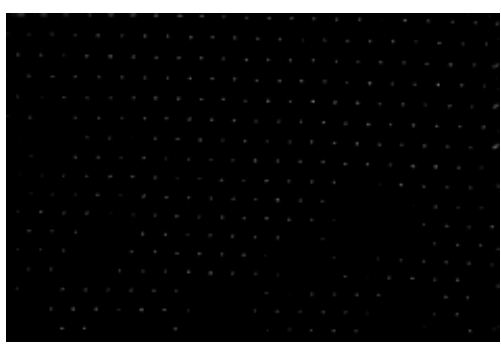

(c) $U H M T_{\mathbf{B}}(f)$.

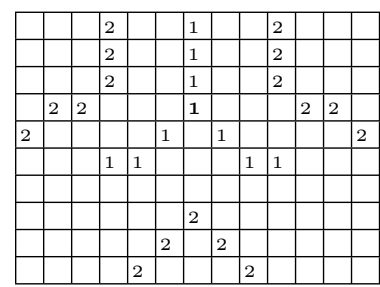

(b) Composite SE $\mathbf{B}$ with pixels of $B_{F G}$ at 1 and $B_{B G}$ at 2 . The origin is the bold 1 pixel.

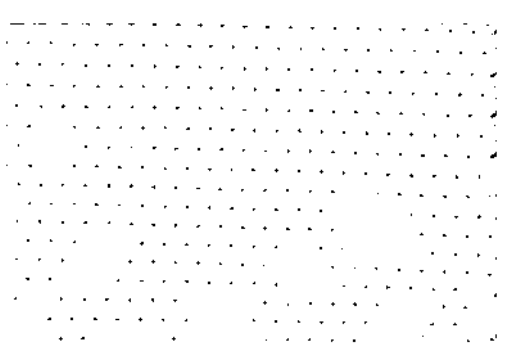

(d) Threshold of $U H M T_{\mathbf{B}}(f)$ for all nonzero values: $T_{t>0}\left[U H M T_{\mathbf{B}}(f)\right]$.

Fig. 4. Grey scale unconstrained hit-or-miss transform extracting the upper corner of each hexagonal cell appearing in the input image. The threshold of the UHMT image for all non-zero values highlights the performance of the detection of the considered surface specific points.

these latter definitions are directly suited to the extension to rank operations, similarly to other morphological transformations [27].

\section{Crest Lines by Order Independent Thinning}

Recent developments about binary order independent homotopic thinnings [16] can be used for extracting crest lines in grey scale images. The principle for anchored grey scale skeletons are detailed in [17] and briefly summarized hereafter. Let us first present a generic definition of an order dependent grey tone thinning algorithm (where $N_{8}^{<}(p)$ denotes the set of neighbours of $p$ whose values are lower than $p)$ :

for each pixel $p$ of $f$

if $p$ is simple in $f$

add $p$ to the set SimpleSet of simple pixels

for each pixel $p \in$ SimpleSet (arbitrary sequential scanning order)

if $p$ is simple in $f$

$$
f(p) \leftarrow \max \left\{f\left(p^{\prime}\right) \mid p^{\prime} \in N_{8}^{<}(p)\right\}
$$


This algorithm will be referred to as Proc1. At the end of Proc1, the input image $f$ contains a thinned image which is homotopic to the original image. Note also that, in accordance with the anti-extensivity property of any thinning, the output image is always less or equal to the input image. Similarly to binary sequential homotopic thinnings, the resulting image depends on the scanning order of the set SimpleSet of simple pixels. This also happens for all algorithms based on grey tone sequential homotopic thinning [10] [26, p. 142] or topological operators $[1,7]$.

An order independent thinning can be obtained as follows [17]: First, apply Proc1 with all possible sequential scanning orders; second, set the value of each pixel $p$ to the maximum output value at $p$. In other words, the output image equals to the point-wise maximum of all possible outputs of Proc1, i.e., for all possible sequential scanning orders of the set SimpleSet of simple pixels. In practice, the notion of order independent thinning allows us to avoid testing all possible configurations by directly testing whether a given simple pixel will be removed by all possible sequential scanning orders. Skeletons are obtained by thinning the image until idempotence. By considering a set of predefined points called anchor points, we obtain anchored skeletons. The algorithm is much faster if each level set of the grey scale image contain at least one anchor point because the Ronse's 8-deletability test of a connected component of simple pixels [18] can be skipped. The extraction of crest lines on a digital elevation model using the regional maxima as anchor points is illustrated in Fig. 5] Note that the resulting skeleton is not one pixel thick and may even contain very thick regions similarly to the thick watersheds described in [34]. This is due to particular configurations of the grey scale values such as those illustrated in Fig. [6 and which has been sampled from Fig. 50 in a region where thick skeletal lines occur. The order independent thinning has set a thick region to the elevation 307. However, this thick region, although mostly surrounded by crest lines, cannot be further thinned (i.e., set to either 1 or 3 in this example) because it is itself leading to a crest line. That is, the pixel appearing in a shaded box is not simple. Thick watershed zones (in the sense that one cannot decide whether the thick region drains to a catchment basin or another) always correspond to thick regions of the grey tone skeleton. Finally, it is worth mentioning that recent work on distance ordered homotopic thinning $[32,15,31]$ could be made independent of the order used for processing the pixels at the current distance value, simply by using order independent thinning of each distance level.

\section{Conclusion and Perspectives}

New developments related to the minima imposition, the lower complete transformation, the hit-or-miss transform, and the extraction of crest lines have been proposed. Beyond practical applications to the processing of Pan-European digital elevation models which are currently investigated [28], we believe that these developments will be of interest to other image analysis problems where the searched features correspond to topographic features. Note that the minima 

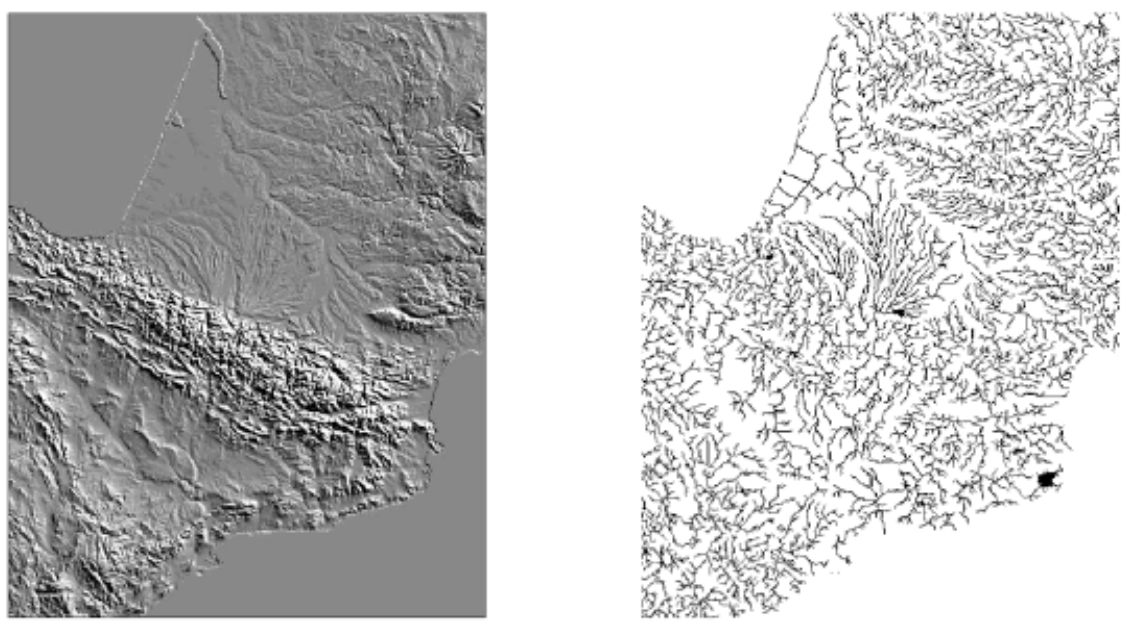

Fig. 5. Crest lines extracted by order independent homotopic thinning. Left: Shaded view of a $1 \mathrm{~km}$ input digital elevation model of the Pyrenees. Right: Support of the skeleton of the DEM using the regional maxima as anchor points.

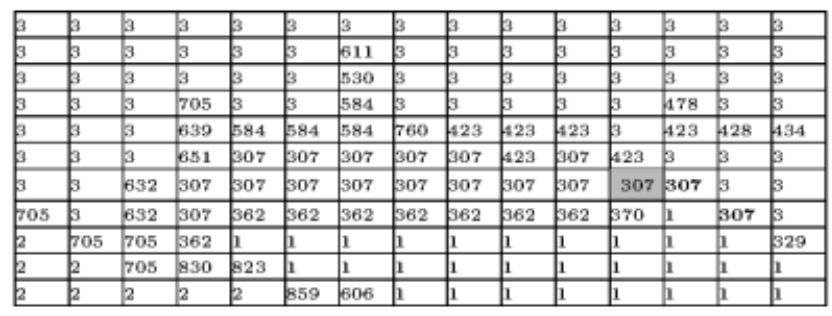

Fig. 6. Thick skeletal lines extracted from Fig. 5 b. The pixels at intensity 1, 2, and 3 belong to the regional minima of the grey tone skeleton, all other pixels belonging to the skeletal lines. The two pixels marked in bold clearly belong to the crest lines, impeding thereby further order independent homotopic thinning of the thick region.

imposition technique based on carving is not incompatible with that based on reconstruction by erosion. Indeed, it is enough to define two sets of markers, the first containing markers leading to a carving while the second to a reconstruction by erosion, the priority queues ensuring an ordered propagation. Finally, it would be of interest to extend the developments of order independent homotopic thinnings to the computation of watersheds and compare this approach with those based on flooding simulations [34], flow simulations [30], and orders [6]. Extensions of the proposed carving, lower complete, and hit-or-miss transformations to 3-D grey scale images are straightforward. However, additional research will be required to extend the notion of order independent homotopic thinnings for producing 3-D grey scale skeletons and watersheds. 


\section{References}

[1] G. Bertrand, J.-C. Everat, and M. Couprie. Image segmentation through operators based upon topology. Journal of Electronic Imaging, 6(4):395-405, 1997.

[2] S. Beucher. Segmentation d'images et morphologie mathématique. PhD thesis, Ecole des Mines de Paris, June 1990.

[3] S. Beucher and F. Meyer. The morphological approach to segmentation: The watershed transformation. In E. Dougherty, editor, Mathematical morphology in image processing, pages 433-481. Marcel Dekker, New York, 1993.

[4] E. Breen and R. Jones. Attribute openings, thinnings, and granulometries. Computer Vision and Image Understanding, 64(3):377-389, 1996.

[5] S. Collins. Terrain parameters directly from a digital elevation model. The Canadian Surveyor, 29(5):507-518, December 1975.

[6] M. Couprie and G. Bertrand. Tesselations by connection in orders. In Proc. of Discrete Geometry for Computer Imagery'2000, Uppsala, volume 1953 of Lecture Notes in Computer Science, pages 15-26. Springer-Verlag, 2000.

[7] M. Couprie, F. Nivando Bezerra, and G. Bertrand. Grayscale image processing using topological operators. In L. Latecki, R. Melter, D. Mount, and A. Wu, editors, Vision Geometry VIII, volume SPIE-3811, pages 261-272, 1999.

[8] H. Digabel and C. Lantuéjoul. Iterative algorithms. In J.-L. Chermant, editor, Quantitative analysis of microstructures in materials sciences, biology and medicine, pages 85-99, Stuttgart, 1978. Dr. Riederer-Verlag GmbH.

[9] J. Garbrecht and L. Martz. The assignment of drainage direction over flat surfaces in raster digital elevation models. Journal of Hydrology, 193:204-213, 1997.

[10] V. Goetcherian. From binary to grey tone image processing using fuzzy logic concepts. Pattern Recognition, 12:7-15, 1980.

[11] R. Haralick. Ridges and valleys on digital images. Computer Vision, Graphics, and Image Processing, 22:28-38, 1983.

[12] D. Mark. Automated detection of drainage networks from digital elevation models. Cartographica, 21:168-178, 1984.

[13] F. Meyer. Skeletons and perceptual graphs. Signal Processing, 16:335-363, 1989.

[14] F. Meyer and S. Beucher. Morphological segmentation. Journal of Visual Communication and Image Representation, 1(1):21-46, September 1990.

[15] C. Pudney. Distance-ordered homotopic thinning: A skeletonization algorithm for 3D digital images. Computer Vision and Image Understanding, 72(3):404413, December 1998.

[16] V. Ranwez and P. Soille. Order Independent Homotopic Thinning. In G. Bertrand, M. Couprie, and L. Perroton, editors, Proc. of Discrete Geometry for Computer Imagery'99, volume 1568 of Lecture Notes in Computer Science, pages 337-346. Springer-Verlag, 1999.

[17] V. Ranwez and P. Soille. Order independent homotopic thinning for binary and grey tone anchored skeletons. Pattern Recognition Letters, 2002, Publication pending.

[18] C. Ronse. A topological characterization of thinning. Theoretical Computer Science, 43:31-41, 1986.

[19] P. Salembier and J. Serra. Flat zones filtering, connected operators, and filters by reconstruction. IEEE Transactions on Image Processing, 4(8):1153-1160, August 1995.

[20] J. Serra. Image analysis and mathematical morphology. Academic Press, London, 1982. 
[21] J. Serra and P. Salembier, editors. Mathematical morphology and its applications to signal processing, 1993. Universitat Politècnica de Catalunya, Barcelona.

[22] P. Soille. Spatial distributions from contour lines: An efficient methodology based on distance transformations. Journal of Visual Communication and Image Representation, 2(2):138-150, June 1991.

[23] P. Soille. Morphologie mathématique: du relief à la dimensionalité - Algorithmes et méthodes-. PhD thesis, Université catholique de Louvain; en collaboration avec l'Ecole des Mines de Paris, February 1992.

[24] P. Soille. Generalized geodesic distances applied to interpolation and shape description. In J. Serra and P. Soille, editors, Mathematical Morphology and its Applications to Image Processing, pages 193-200. Kluwer Academic Publishers, 1994

[25] P. Soille. Generalized geodesy via geodesic time Pattern Recognition Letters, 1235-1240, December 1994

[26] P. Soille. Morphological image analysis: Principles and Applications. SpringerVerlag, Berlin, New York, 1999. Second extended edition to appear in 2002, see also http://ams.egeo.sai.jrc.it/soille

[27] P. Soille. On morphological operators based on rank filters. Pattern Recognition,2002.

[28] P. Soille. Carving and adpative drainage enforcement of grid digital elevation models. Water Resources Research, Submitted.

[29] P. Soille and M. Ansoult. Automated basin delineation from digital elevation models using mathematical morphology. Signal Processing, 20: 171-182, June 1990.

[30] P. Soille and C. Gratin. An efficient algorithm for drainage networks extraction on DEMs. Journal of Visual Communication and Image Representation, 5(2): 181-189, June 1994.

[31] S. Svensson, G. Borgefors, and I. Nyström. On reversible skeletonization using anchor-points from distance transforms. Journal of Visual Communication and Image Representation, 10:379-397, 1999.

[32] H. Talbot and L. Vincent. Euclidean skeletons and conditional bisectors. In P. Maragos, editor, Visual Communications and Image Processing, volume SPIE1818, pages 862-876, 1992.

[33] B. Verwer, P. Verbeek, and S. Dekker. An efficient cost algorithm applied to distance transforms. IEEE Transactions on Pattern Analysis and Machine Intelligence, 11(4):425-429, April 1989.

[34] L. Vincent and P. Soille. Watersheds in digital spaces: An efficient algorithm based on immersion simulations. IEEE Transactions on Pattern Analysis and Machine Intelligence, 13(6):583-598, June 1991. 